\title{
Mechanistic diversity in ATP-binding cassette (ABC) transporters
}

\section{Review Article}

Author(s):

Locher, Kaspar P.

Publication date:

2016

Permanent link:

https://doi.org/10.3929/ethz-b-000117629

Rights / license:

In Copyright - Non-Commercial Use Permitted

Originally published in:

Nature Structural \& Molecular Biology 23(6), https://doi.org/10.1038/nsmb.3216 


\title{
Mechanistic diversity in ATP-binding cassette (ABC) transporters
}

Kaspar P. Locher

Institute of Molecular Biology and Biophysics, Department of Biology, ETH Zurich.

\begin{abstract}
$A B C$ transporters catalyze transport reactions ranging from high-affinity uptake of micronutrients into bacteria to the extrusion of cytotoxic compounds from mammalian cells. Crystal structures of $A B C$ domains and full transporters have provided a framework for formulating reaction mechanisms of ATP-driven substrate transport, but recent studies suggest a remarkable mechanistic diversity within the protein family. This review evaluates the differing mechanistic proposals and outlines future directions to explore $A B C$ transporter-catalyzed reactions.
\end{abstract}

\section{Introduction}

$A B C$ transporters are a family of membrane proteins that mediate diverse, ATP-driven transport processes. They contain a pair of conserved, cytoplasmic domains termed ATP-binding cassettes $(A B C)$ or nucleotide-binding domains (NBD). NBDs hydrolyze ATP and drive conformational changes in the attached transmembrane domains (TMDs), allowing substrates to cross the lipid bilayer of the membrane either into the cytoplasm (import) or out (export). The domain arrangement and conserved sequence motifs are summarized in box 1 , and representative structures of the different sub-families of $A B C$ transporters are shown in Fig. 1.

$A B C$ transporters are found in all domains of life. In bacteria, $A B C$ importers are involved in the uptake of nutrients and micro-nutrients through medium- and high-affinity pathways ${ }^{1,2}$. These reactions are highly specific and require a periplasmic (or lipid-anchored external) substrate binding protein $^{3,4}$. Bacterial ABC exporters have diverse roles, including the extrusion of (often lipid-linked) building blocks required for cell wall assembly ${ }^{5-7}$. Other $A B C$ exporters are poly-specific and contribute to drug resistance by exporting certain toxic substances ${ }^{8,9}$.

There are 48 distinct human $A B C$ transporters that belong to various sub-families ${ }^{10}$. Most are thought to be exporters, and some display a remarkable poly-specificity for substrates. Some human ABC transporters have functions other than substrate translocation, e.g. in regulating potassium channels (SUR1 and SUR2) $^{11,12}$ or acting as an ATP-gated chloride channel (CFTR) $)^{13}$. Many human ABC transporters are bio-medically and clinically relevant: For example, multidrug transporters such as $A B C B 1, A B C G 2$, or $A B C C 1$ protect various organs from toxic insult, but also contribute to drug resistance of cancer cells ${ }^{14-17}$; In liver, a series of $A B C$ transporters (ABCB11, ABCB4, ABCG5/G8) catalyzes the generation of bile, with dysfunction of either transporter leading to liver disease ${ }^{18-22}$; The transporter of antigenic peptides (TAP, a heterodimer of $A B C B 2$ and $A B C B 3$ ) has an important role in the adaptive immune response ${ }^{23,24}$. Archaea, yeasts, plants, and human parasites also have $A B C$ transporters that catalyze vital functions (reviewed in ${ }^{25-27}$ ).

In addition to the fundamental question of how they translocate large substrates across lipid bilayers, a detailed structural and mechanistic understanding of $A B C$ transporters may contribute to drug discovery, for example in the case of multidrug or liver $A B C$ transporters ${ }^{28-31}$.

It has been established that the closed NBD dimer conformation is essential for ATPase activity of full $A B C$ transporters, because physical NBD separation results in the transporter inactivation ${ }^{32-34}$. Given the tight interaction between the NBDs and the coupling helices, it is generally accepted that the conserved power stroke of $A B C$ transporters is the pushing together and pulling apart of the 
cytoplasmic ends of the TMDs during the ATP hydrolysis cycle (reviewed in ${ }^{35-38}$ ). However, recent structural and mechanistic studies challenged the notion that a single, conserved mechanism can explain the reactions even within individual sub-families of $A B C$ transporters. This review evaluates transport mechanisms derived from structures of full transporters as well as from functional, spectroscopic, and biophysical studies.

\section{Alternating access in $A B C$ importers}

Type I ABC importers. Type I ABC importers facilitate medium-affinity uptake of diverse nutrients including ions, sugars, amino acids, short peptides, and oligosaccharides, into bacteria. The E. coli maltose transporter has been extensively studied (REFS) and can serve as a mechanistic model of a generic mechanism of type $1 \mathrm{ABC}$ importers (Fig. 2a). The transport cycle can be thought to start in an inward-facing conformation, with substrate-loaded binding protein approaching the external side of the transporter. There is a possibility of futile ATPase activity at this state, resulting in a "basal ATPase rate". However, this activity is slow in type 1 ABC importers and often additionally inhibited. Docking of the substrate binding protein stimulates the basal ATPase rate of the maltose ${ }^{39}$ and alginate transporters ${ }^{40}$, but not of the molybdate/tungstate transporters ${ }^{32,41}$. The key conformational transition during the transport cycle (state 1 to 2 ) is the closing of the NBDs, which pushes the coupling helices towards each other and converts the TMDs to an outward-facing conformation. This generates a tunnel from the attached binding protein to a low-affinity substrate-binding pocket in the translocation pathway, halfway across the membrane. In the maltose transporter, two effects were found to ensure the release of substrate from the binding protein: First, the binding pocket is slightly distorted. Second, periplasmic TMD loops (in particular a "scoop loop") cause a partial steric clash with bound substrate ${ }^{42}$. Although the affinity of maltose to the temporary binding site between the TMDs is only moderate ( $\mathrm{Kd}$ in the millimolar range), the substrate populates this pocket because the local concentration in the tunnel is at least 2 orders of magnitude above the dissociation constant. In the subsequent, irreversible step (state 2 to 3 ) ATP is hydrolyzed and inorganic phosphate released. This causes the NBD dimer to open, pulling the coupling helices outwards and triggering the conversion to an inward-facing conformation. The substrate can now dissociate into the cytoplasm. Finally, the exchange of ADP for ATP resets the system (state 3 to 1).

There are mechanistic variations of the theme in certain type $1 A B C$ importers. Because of their role in medium-affinity pathways, efficient coupling of ATP hydrolysis to transport is important, and the observed basal ATPase activities are generally low. While some transporters such as OpuA have strict coupling of 2 ATP per transport cycle ${ }^{43}$, additional regulatory strategies have been observed for other transporters in the form of trans-inhibition ${ }^{32,33,44}$ or the binding of regulatory proteins ${ }^{45}$.

Type 2 ABC importers. These are generally part of high-affinity uptake pathways for metal chelates including heme and other iron-containing complexes, and cobalamin (reviewd in ${ }^{46,47}$ ). These substrates are not only larger and more hydrophobic than those of type I ABC importers, but available at low concentrations only. The mechanism proposed for the E. coli vitamin B12 transporter BtuCD-F can serve as a model for other type 2 ABC importers. Unlike observed in the maltose transporter, there is no measurable affinity (and thus no substrate binding pocket) for B12 in the TMDs of BtuCD$\mathrm{F}$. The obtained crystal structures revealed three gate regions in the transmembrane BtuC subunits, two on the cytoplasmic side (cyto-gates 1 and 2 ) and one on the periplasmic side (peri-gate) $)^{48,49}$. The key difference to type $1 \mathrm{ABC}$ importers is that in BtuCD-F, ATP binding and hydrolysis does not generate an inward-facing conformation by itself. Rather, the two cytoplasmic gates separate the cytoplasm from the central translocation cavity both in the ATP-bound and nucleotide-free states, as long as transport substrate is absent. Figure $3 \mathrm{~b}$ shows a schematic of the transport mechanism deduced from structural, biochemical, and spectroscopic data ${ }^{48-52}$. The cycle can be thought to start in 
an ATP-bound state (state 1) with a closed NBD dimer conformation, and coupling helices pushed together, which causes cyto-gate 2 to close. Because the peri-gate is open, state 1 is an outwardfacing conformation. Futile ATPase activity (arrows to the left) has been observed in all type $2 \mathrm{ABC}$ importers studied to date, although the measured rates are very low and the loss of cytoplasmic ATP apparently manageable for the cell. State 2 is reached upon docking of the substrate binding protein and the release of substrate into a hydrophobic ("teflon") cavity with no measurable affinity. As in type 1 importers, the high-affinity pocket in the substrate binding protein is distorted upon docking, and loops of the TMD cause a steric clash, "scooping" the substrate from its pocket and enclosing it in the translocation pathway between the TMDs. In BtuCD, trapping of substrate in this cavity could only be demonstrated in liposomes. In detergent, the substrate escapes from the cavity, probably due to increased protein dynamics or competition for binding with detergent molecules. The following, irreversible step (state 2 to 3 ) is the hydrolysis of ATP and the release of phosphate, which requires (at least partial) opening of the NBD dimer, pulling the coupling helices apart and opening cyto-gate 2. Due to the size of the trapped transport substrate, cyto-gate 1 is now unable to close, which probably causes a strained conformation and pressure from the sides onto the substrate. This resembles a peristaltic force and may contribute to substrate dissociation into the cytoplasm. Upon substrate release, BtuCD relaxes into an asymmetric conformation (state 4) that does not feature a central cavity anymore, because both cyto-gate 1 and the peri-gate are now closed ${ }^{51}$. This state is very stable in vitro and probably probably prevents potential ion leakage during the subsequent re-setting of the system. The asymmetric conformation of state 4 appears unique to type 2 importers because the structural elements involved are not present in type 1 importers. Notably, the mechanistic proposal does not assign any role to the BtuCD conformation first observed, an apo-state with outward-facing TMDs $^{53}$. Although such a conformation may transiently occur in the cell (before ADP is replaced by ATP) and contribute to futile ATP hydrolysis, it is not essential to formulate a productive transport cycle. Another important point is that nucleotide-bound BtuCD with a closed NBD dimer could only be stably trapped and crystallized upon introduction of a disulfide cross-link that covalently linked the Dloops of the two NBDs.

\section{Distinct mechanistic proposals for B-family ABC exporters}

Despite a number of high-resolution structures reported, no common mechanism has been established for B-family ABC exporters. One problem is that most structures do not reveal bound substrates, a consequence of their hydrophobicity and low binding affinity in detergent solution. Another issue is that when removed from the membrane and in the absence of ATP (or ATP-ADP mixtures reflecting average cytoplasmic concentrations), B-family $A B C$ exporters are prone to adopting inward-facing conformations with occasionally very large separations of the NBDs. When bound to nucleotides, the conformations tend to show a smaller separation of the NBDs as well as occluded or outward-facing conformations. Figure 3 shows a selection of structures of B-family $A B C$ exporters, demonstrating the wide conformational range.

Early mechanistic proposals stated that alternating access in B-family $A B C$ exporters is reached by converting the observed, inward-facing conformations to outward-facing states with bound ATP. However, several crystal structures are inconsistent with this simplified view ${ }^{54-58}$. The relevance of the observed inward-facing conformations has therefore been controversially discussed (see box 2), and the discussion is not simply about semantics. The critical issue is whether an observed structure reflects a functionally relevant state. If it is concluded that a conformation is not physiologically relevant (as were the inward-facing conformation of the LLO flippase PgIK), there would be no point in attempting to dock a transport substrates into the observed cavities. The two extreme views outlined in box 2 could be reconciled by viewing B-family $A B C$ exporters as springs, whose architecture works to push their NBDs apart. In cellular membranes, the combined effects of lipids, ATP, and substrate 
would allow the transporters to reach outward-facing conformations. Inward-facing conformations might reflect physiological states if they provide substrate binding sites or may occur transiently because the NBD dimer needs to release the ATP hydrolysis products. Inward-facing conformations without NBD contact or substrate binding pockets are likely unphysiological.

As was observed for ABC importers, substrates and inhibitors can modulate the ATPase activity of Bfamily $A B C$ exporters. The molecular basis of this phenomenon is unknown, but stimulation or inhibition is often used to identify specific interactions. Two distinct mechanisms of B-family $A B C$ exporters are shown in Figure 4.

Alternating access. An essential component of this mechanistic proposal (Fig. 4a) is the binding of transport substrate to an inward-facing pocket of the TMDs (state 1). The relevant binding site may not only be accessible from the cytoplasm, but also from the inner leaflet of the lipid bilayer. Highly hydrophobic substrates (drugs, phospholipids) could thus be directly recruited from within the membrane. The inward-facing conformation is linked to an open NBD conformation. As for ABC importers, futile ATP hydrolysis may occur in the absence of substrate, albeit at a slower rate than when substrate is bound. For poly-specific transporters (including the multidrug transporter ABCB1), it is unclear if there is only one binding pocket ${ }^{59}$, as some findings suggest multiple sites ${ }^{60,61}$. In the case of TAP, it was concluded that the $\mathrm{N}$ - and C-termini of all transported peptides are firmly bound, whereas the central aminoacids may only weakly interact with the transporter ${ }^{62}$. Once a substrate is recruited, the transporter probably converts to an occluded conformation (state 1 to 2), with ATP bound between closed NBDs, but without access to the central cavity from either side of the membrane. Such a conformation was observed in the structures of AMPPNP-bound McjD ${ }^{63}$ and ATP $\gamma$ S-bound PCAT ${ }^{57}$, albeit without bound substrate. It is unclear if the occluded conformation is a mandatory intermediate. The following step (state 2 to 3 ) reflects the conversion to the outward-facing conformation, allowing substrate release into the external medium or into the outer leaflet of the lipid bilayer. Given that there is a re-arrangement of the TM helices from states 1 to 3 , with different sets of helices surrounding the translocation pathway and forming the two external wings (Fig. 4), there is a change in the cavity surface surrounding the substrate. In the AMPPNP-bound, outward-facing Sav1866 structure, a rather hydrophilic cavity was observed, suggesting that a chemical mismatch to the hydrophobic drugs could be driving substrate release ${ }^{64}$. This notion was in line with observations that the apparent affinity of multidrug $A B C$ transporters for substrates was lower from the external than from the cytoplasmic side ${ }^{65}$. If a substrate is highly hydrophobic (such as in the case of certain tumor drugs or phospholipids), it is likely to re-partition into the membrane and may only remain in the external medium when bound to a carrier protein (e.g. BSA) and swept away in the blood stream, or if stabilized in a mixed micelle such as in bile. In the final step (state 3 to 1), hydrolysis of ATP and the release of inorganic phosphate drives the NBDs apart and, by transmitting this motion to the TMDs via the coupling helices, converts the outward-facing into an inward-facing conformation. This step is irreversible given the large amount of energy gained from the hydrolysis of ATP (approaching 50kJ $\mathrm{mol}^{-1}$ for one ATP). It is unclear if the hydrolysis of two ATP molecules is simultaneous or consecutive, nor is it certain that both ATP molecules are hydrolyzed during every transport cycle (see below).

Outward-only mechanism. This mechanism was proposed for the LLO flippase PglK ${ }^{66}$ (Fig. 4b). As its name implies, the outward-only mechanism does not invoke an inward-facing cavity to interact with the substrate. Although two distinct inward-facing conformations were determined for PglK, the observed cavities did not appear to contribute to the interaction with the substrate. The cycle of the outward-only mechanism can be thought to start in an ATP-bound state and an outward-facing TMD conformation (state 1). Whereas the lipidic polyprenyl tail of the substrate probably remains attached to the lipid-facing surface of the transporter, the pyrophosphate-glycan head-group is proposed to be transferred directly into the outward-facing cavity, where strong, electrostatic interactions between 
arginines and the pyrophosphate group are formed (state 1 to 2). Once the head-group is bound in the outward-facing cavity, ATP hydrolysis and the release of inorganic phosphate drives the NBDs apart and the external wings of the TMDs together (state 3). The extrusion of the substrate appears essential to avoid a steric clash, similar to the peristaltic component proposed for type $2 A B C$ importers. The important concept is that upon release of the LLO head-group, the transporter adopts a conformation with insufficient size to fit a substrate. In the final step (state 3 to 1 ), dissociation of the substrate, re-binding of a new substrate, and exchange of ADP for ATP resets the system. Unlike the alternating-access mechanism, the outward-only mechanism does not rely on a large separation of NBDs, although a certain opening of the sandwich dimer is required for phosphate release and nucleotide exchange. The mechanism appears suitable to rationalize not only the flipping of LLOs, but the export of very large substrates (e.g. proteins or long polysaccharides, see also below), as repeated ratcheting through inward-facing conformations while constantly hydrolyzing ATP molecules appears sterically impossible. It is particularly attractive to consider the outward-only mechanism not only for lipid-linked oligosaccharides, but also for phospholipid and cholesterol export (transported by ABCB4, ABCG5/G8, and potentially ABCG1 and ABCA1).

It should be noted that the alternating access and outward-only mechanisms are not mutually exclusive and may be used by a single transporter for different substrates. For example, one could imagine that a multidrug $A B C$ transporter might employ the alternating-access mechanism for certain substrates while using the outward-only mechanism for others.

The mechanistic proposals shown in Fig. 4 are generic and there are multiple variations and complications. Arguably the most important is the fact that some B-family ABC exporters contain only one consensus ATP binding site, while the other site is formed by degenerate motifs that reduce or abolish ATP binding or hydrolysis at that site. Transporters with published structures include Tm287/8, $\mathrm{TmrAB}$, and $\mathrm{TAP}^{55,56,67,68}$. Of those, $\mathrm{Tm} 287 / 8$ has been resolved at sufficiently high resolution to allow side chain placement, and although a coupling of the ATPase sites via the D-loops was proposed, a definitive role of the asymmetry for the transport mechanism was not established ${ }^{55,56}$. EPR studies comparing a homodimeric $A B C$ exporter (MsbA, containing two functional ATPase sites) with a heterodimeric one (BmrAB, containing one functional, one degenerate site) concluded that the presence of a degenerate ATPase site may prevent a wide opening of the NBDs in BmrAB, possibly indicating a fundamental difference between symmetrical vs. asymmetrical transporters ${ }^{69}$. It should be noted that ATPase asymmetry may not be restricted to heterodimeric $A B C$ transporters. Even $A B C$ transporters with two functional, consensus ATPase sites may reach an asymmetric state s0-72 $^{70}$ even hydrolyze only one ATP molecule per transport cycle. The asymmetry of ATPase sites extends beyond the $B$-family of $A B C$ transporters: The cystic fibrosis transmembrane conductance regulator $(A B C C 7)$ also features a degenerate site in addition to the consensus site and it is currently assumed that binding of ATP to the degenerate site of CFTR is essential for chloride conductance, even though nucleotide is only hydrolyzed at the consensus site ${ }^{73}$. Another detail omitted in Fig. 4 is the role of the D-loops: A replacement of the conserved $D$-loop aspartates was found to convert TAP from an active transporter into a passive facilitator, suggesting a role in the coupling of ATP hydrolysis to vectorial transport $^{74}$. The structural basis of this observation remains to be established, and it is currently unknown if other $\mathrm{ABC}$ transporters have similar phenotypes when their D-loops are mutated.

\section{Mechanisms: Does one size fit all?}

A number of attempts have been made to formulate generally applicable mechanisms for $A B C$ transporters. These include, among others, the "switch model", the "constant contact model", or the "reciprocating twin-channel model" 38,75 . However, given the structural and biochemical differences discussed above, mechanistic similarities in the entire superfamily appear restricted to the events at 
the NBDs. This is in line with the observation that not all $A B C$ transporters have the same phylogenetic origin ${ }^{76,77}$. Rather, distinct TMD folds appear to have evolved separately. Because the different TMDs all feature coupling helices, they can interact with NBDs to harness the conformational power provided by ATP hydrolysis and facilitate import or export in a given ABC transporter. The TMDs contain distinct binding sites adapted to recognize substrates of highly diverse mass and chemical nature and feature gates at different locations in the TMDs. In conclusion: No one size fits all. Rather than attempting to formulate unified transport mechanisms, I believe the goal must be to define the specifics of every $A B C$ system under study. This requires a combination of structural, biochemical, and biophysical (including single molecule spectroscopic ${ }^{78}$ ) studies, and ultimately computational analysis, which, despite progress with select systems ${ }^{79-82}$, has this far struggled with the fact that $A B C$ transporters are very slow enzymes, with turnover rates approaching seconds in some cases.

\section{ABC transporter research: Golden days ahead}

Significant progress in understanding reaction mechanisms has been made since the first structures of $A B C$ transporters were determined. However, many details of how these transporters work remain to be discovered, and this search may yield surprises and unexpected findings even for B-family exporters. For example, new mechanistic concepts may have to be established for rationalizing phospholipid flipping by $A B C B 4$. In addition, there are structurally uncharacterized $A B C$ transporters that will likely reveal new TMD folds. These include the human G-family ABC transporters, but also bacterial $A B C$ exporters of capsular polysaccharides ${ }^{83}$.

Some $A B C$ transporters are part of larger cellular assemblies. For example, TAP is part of the endoplasmatic peptide loading complex, where protein-protein interactions control and influence the transport activity. SUR1 and SUR2 (ABCC8 and ABCC9) form complexes with inward-rectifying potassium channels ${ }^{12}$, generating assemblies approaching a megadalton in mass and featuring almost a dozen ATP-binding sites for fine regulation of channel activity. In bacteria, there are $A B C$ transporters involved in the secretion of toxic hemolysins ${ }^{84}$, which is achieved by assembly of an $A B C$ transporter (HlyB) with periplasmic and outer membrane components (HlyD and TolC). To gain insight into these larger assemblies, single particle cryo-electron microscopy will likely be indispensible. However, given the recent resolution revolution in single particle cryo-EM ${ }^{85}$, even single $A B C$ transporters may soon be studied at high resolution without the need to grow three-dimensional crystals. At present, the resolution of the cryo-EM structures of two $A B C$ transporters is in the range of $6-8 \AA$ (TmrAB, Tap $)^{67,68}$, but further developments in technology and sample preparation may soon allow resolutions sufficient for side chain placement to be obtained. This will have a large impact on the field, as transport intermediates and samples that have not yielded to crystallization efforts may be visualized, allowing biochemists and structural biologists to probe further into the intricacies of $A B C$ transporter reactions. It's an exciting time indeed for $A B C$ transporter research.

Acknowledgements: The author thanks Amer Alam and Camilo Perez for critical reading of the manuscript. Research in the author's lab has been supported by funds from ETH Zurich, the Swiss National Science Foundation (SNF), the Roche Research Fund, the Swiss Cancer League Oncosuisse, the National Centers for Excellence in Research (NCCR) Structural Biology and TransCure, and EMBO. 


\section{References}

1. Davidson, A.L., Dassa, E., Orelle, C. \& Chen, J. Structure, function, and evolution of bacterial ATP-binding cassette systems. Microbiology and Molecular Biology Reviews 72, 317-364 (2008).

2. Cui, J.M. \& Davidson, A.L. ABC solute importers in bacteria. Essays in Biochemistry: Abc Transporters 50, 85-99 (2011).

3. Berntsson, R.P.A., Smits, S.H.J., Schmitt, L., Slotboom, D.J. \& Poolman, B. A structural classification of substrate-binding proteins. Febs Letters 584, 2606-2617 (2010).

4. Maqbool, A. et al. The substrate-binding protein in bacterial ABC transporters: dissecting roles in the evolution of substrate specificity. Biochemical Society Transactions 43, 1011-1017 (2015).

5. Raetz, C.R.H., Reynolds, C.M., Trent, M.S. \& Bishop, R.E. Lipid a modification systems in gramnegative bacteria. Annual Review of Biochemistry 76, 295-329 (2007).

6. Cuthbertson, L., Kos, V. \& Whitfield, C. ABC Transporters Involved in Export of Cell Surface Glycoconjugates. Microbiology and Molecular Biology Reviews 74, 341-+ (2010).

7. Ruiz, N., Gronenberg, L.S., Kahne, D. \& Silhavy, T.J. Identification of two inner-membrane proteins required for the transport of lipopolysaccharide to the outer membrane of Escherichia coli. Proceedings of the National Academy of Sciences of the United States of America 105, 55375542 (2008).

8. Wong, K., Ma, J., Rothnie, A., Biggin, P.C. \& Kerr, I.D. Towards understanding promiscuity in multidrug efflux pumps. Trends in Biochemical Sciences 39, 8-16 (2014).

9. Seeger, M.A. \& van Veen, H.W. Molecular basis of multidrug transport by ABC transporters. Biochimica Et Biophysica Acta-Proteins and Proteomics 1794, 725-737 (2009).

10. Moitra, K. \& Dean, M. Evolution of ABC transporters by gene duplication and their role in human disease. Biological Chemistry 392, 29-37 (2011).

11. Bryan, J. et al. $A B C C 8$ and $A B C C 9$ : $A B C$ transporters that regulate $\mathrm{K}(+)$ channels. Pflugers Archiv-European Journal of Physiology 453, 703-718 (2007).

12. Aittoniemi, J. et al. SUR1: a unique ATP-binding cassette protein that functions as an ion channel regulator. Philosophical Transactions of the Royal Society B-Biological Sciences 364, 257-267 (2009).

13. Riordan, J.R. CFTR function and prospects for therapy. Annual Review of Biochemistry 77, 701726 (2008).

14. Sarkadi, B., Homolya, L., Szakacs, G. \& Varadi, A. Human multidrug resistance ABCB and ABCG transporters: Participation in a chemoimmunity defense system. Physiological Reviews 86, 11791236 (2006).

15. Gottesman, M.M., Fojo, T. \& Bates, S.E. Multidrug resistance in cancer: Role of ATP-dependent transporters. Nature Reviews Cancer 2, 48-58 (2002).

16. Sharom, F.J. The P-glycoprotein multidrug transporter. Essays in Biochemistry: Abc Transporters 50, 161-178 (2011).

17. Gillet, J.P. \& Gottesman, M.M. Advances in the Molecular Detection of ABC Transporters Involved in Multidrug Resistance in Cancer. Current Pharmaceutical Biotechnology 12, 686-692 (2011).

18. Dawson, S., Stahl, S., Paul, N., Barber, J. \& Kenna, J.G. In Vitro Inhibition of the Bile Salt Export Pump Correlates with Risk of Cholestatic Drug-Induced Liver Injury in Humans. Drug Metabolism and Disposition 40, 130-138 (2012).

19. Morita, S.Y. \& Terada, T. Molecular Mechanisms for Biliary Phospholipid and Drug Efflux Mediated by ABCB4 and Bile Salts. Biomed Research International (2014).

20. Nicolaou, M. et al. Canalicular ABC transporters and liver disease. Journal of Pathology 226, 300315 (2012).

21. Linton, K.J. Lipid flopping in the liver. Biochemical Society Transactions 43, 1003-1010 (2015).

22. Stieger, B. Role of the bile salt export pump, BSEP, in acquired forms of cholestasis. Drug Metabolism Reviews 42, 437-445 (2010).

23. Abele, R. \& Tampe, R. The TAP translocation machinery in adaptive immunity and viral escape mechanisms. Essays in Biochemistry: Abc Transporters 50, 249-264 (2011).

24. Eggensperger, S. \& Tampe, R. The transporter associated with antigen processing: a key player in adaptive immunity. Biological Chemistry 396, 1059-1072 (2015).

25. Leprohon, P., Legare, D. \& Ouellette, M. ABC transporters involved in drug resistance in human parasites. Essays in Biochemistry: Abc Transporters 50, 121-144 (2011). 
26. Rea, P.A. Plant ATP-Binding cassette transporters. Annual Review of Plant Biology 58, 347-375 (2007).

27. Martinoia, E. et al. Multifunctionality of plant $A B C$ transporters - more than just detoxifiers. Planta 214, 345-355 (2002).

28. Tamaki, A., lerano, C., Szakacs, G., Robey, R.W. \& Bates, S.E. The controversial role of ABC transporters in clinical oncology. Essays in Biochemistry: Abc Transporters 50, 209-232 (2011).

29. Morgan, R.E. et al. A Multifactorial Approach to Hepatobiliary Transporter Assessment Enables Improved Therapeutic Compound Development. Toxicological Sciences 136, 216-241 (2013).

30. Palmer, A.M. \& Alavijeh, M.S. Overview of experimental models of the blood-brain barrier in CNS drug discovery. Current protocols in pharmacology / editorial board, S.J. Enna 62, Unit 715 (2013).

31. Montanari, F. \& Ecker, G.F. Prediction of drug-ABC-transporter interaction - Recent advances and future challenges. Advanced Drug Delivery Reviews 86, 17-26 (2015).

32. Gerber, S., Comellas-Bigler, M., Goetz, B.A. \& Locher, K.P. Structural basis of trans-inhibition in a molybdate/tungstate ABC transporter. Science 321, 246-250 (2008).

33. Kadaba, N.S., Kaiser, J.T., Johnson, E., Lee, A. \& Rees, D.C. The high-affinity E-coli methionine ABC transporter: Structure and allosteric regulation. Science 321, 250-253 (2008).

34. Ward, A.B. et al. Structures of P-glycoprotein reveal its conformational flexibility and an epitope on the nucleotide-binding domain. Proceedings of the National Academy of Sciences of the United States of America 110, 13386-13391 (2013).

35. Dawson, R.J.P., Hollenstein, K. \& Locher, K.P. Uptake or extrusion: crystal structures of full ABC transporters suggest a common mechanism. Molecular Microbiology 65, 250-257 (2007).

36. Oldham, M.L., Davidson, A.L. \& Chen, J. Structural insights into ABC transporter mechanism. Current Opinion in Structural Biology 18, 726-733 (2008).

37. Kerr, I.D., Jones, P.M. \& George, A.M. Multidrug efflux pumps: The structures of prokaryotic ATPbinding cassette transporter efflux pumps and implications for our understanding of eukaryotic Pglycoproteins and homologues. Febs Journal 277, 550-563 (2010).

38. George, A.M. \& Jones, P.M. Perspectives on the structure-function of ABC transporters: The Switch and Constant Contact Models. Progress in Biophysics \& Molecular Biology 109, 95-107 (2012).

39. Chen, J., Sharma, S., Quiocho, F.A. \& Davidson, A.L. Trapping the transition state of an ATPbinding cassette transporter: Evidence for a concerted mechanism of maltose transport.

Proceedings of the National Academy of Sciences of the United States of America 98, 1525-1530 (2001).

40. Maruyama, Y. et al. Structure of a Bacterial ABC Transporter Involved in the Import of an Acidic Polysaccharide Alginate. Structure 23, 1643-1654 (2015).

41. Hollenstein, K., Frei, D.C. \& Locher, K.P. Structure of an ABC transporter in complex with its binding protein. Nature 446, 213-216 (2007).

42. Oldham, M.L., Khare, D., Quiocho, F.A., Davidson, A.L. \& Chen, J. Crystal structure of a catalytic intermediate of the maltose transporter. Nature 450, 515-U7 (2007).

43. Patzlaff, J.S., van der Heide, T. \& Poolman, B. The ATP/Substrate stoichiometry of the ATPbinding cassette (ABC) transporter OpuA. Journal of Biological Chemistry 278, 29546-29551 (2003).

44. Yang, J.G. \& Rees, D.C. The Allosteric Regulatory Mechanism of the Escherichia coli MetNI Methionine ATP Binding Cassette (ABC) Transporter. Journal of Biological Chemistry 290, 91359140 (2015).

45. Chen, S.S., Oldham, M.L., Davidson, A.L. \& Chen, J. Carbon catabolite repression of the maltose transporter revealed by X-ray crystallography. Nature 499, 364-U147 (2013).

46. Braun, V. \& Hantke, K. Recent insights into iron import by bacteria. Current Opinion in Chemical Biology 15, 328-334 (2011).

47. Koster, W. ABC transporter-mediated uptake of iron, siderophores, heme and vitamin B12. Research in microbiology 152, 291-301 (2001).

48. Korkhov, V.M., Mireku, S.A. \& Locher, K.P. Structure of AMP-PNP-bound vitamin B-12 transporter BtuCD-F. Nature 490, 367-+ (2012).

49. Korkhov, V.M., Mireku, S.A., Veprintsev, D.B. \& Locher, K.P. Structure of AMP-PNP-bound BtuCD and mechanism of ATP-powered vitamin B12 transport by BtuCD-F. Nature Structural \& Molecular Biology 21, 1097-1099 (2014). 
50. Goetz, B.A., Perozo, E. \& Locher, K.P. Distinct gate conformations of the ABC transporter BtuCD revealed by electron spin resonance spectroscopy and chemical cross-linking. Febs Letters 583, 266-270 (2009).

51. Hvorup, R.N. et al. Asymmetry in the structure of the $A B C$ transporter-binding protein complex BtuCD-BtuF. Science 317, 1387-1390 (2007).

52. Joseph, B., Jeschke, G., Goetz, B.A., Locher, K.P. \& Bordignon, E. Transmembrane Gate Movements in the Type II ATP-binding Cassette (ABC) Importer BtuCD-F during Nucleotide Cycle. Journal of Biological Chemistry 286, 41008-41017 (2011).

53. Locher, K.P., Lee, A.T. \& Rees, D.C. The E-coli BtuCD structure: A framework for ABC transporter architecture and mechanism. Science 296, 1091-1098 (2002).

54. Dawson, R.J.P. \& Locher, K.P. Structure of a bacterial multidrug ABC transporter. Nature 443, 180-185 (2006).

55. Hohl, M., Briand, C., Grutter, M.G. \& Seeger, M.A. Crystal structure of a heterodimeric ABC transporter in its inward-facing conformation. Nature Structural \& Molecular Biology 19, 395-402 (2012).

56. Hohl, M. et al. Structural basis for allosteric cross-talk between the asymmetric nucleotide binding sites of a heterodimeric ABC exporter. Proceedings of the National Academy of Sciences of the United States of America 111, 11025-11030 (2014).

57. Lin, D.Y.W., Huang, S. \& Chen, J. Crystal structures of a polypeptide processing and secretion transporter. Nature 523, 425-U92 (2015).

58. Shintre, C.A. et al. Structures of ABCB10, a human ATP-binding cassette transporter in apo- and nucleotide-bound states. Proceedings of the National Academy of Sciences of the United States of America 110, 9710-9715 (2013).

59. Loo, T.W. \& Clarke, D.M. Mutational analysis of ABC proteins. Archives of Biochemistry and Biophysics 476, 51-64 (2008).

60. Chufan, E.E. et al. Multiple Transport-Active Binding Sites Are Available for a Single Substrate on Human P-Glycoprotein (ABCB1). Plos One 8(2013).

61. McCormick, J.W., Vogel, P.D. \& Wise, J.G. Multiple Drug Transport Pathways through Human PGlycoprotein. Biochemistry 54, 4374-4390 (2015).

62. Herget, $M$. et al. Conformation of peptides bound to the transporter associated with antigen processing (TAP). Proceedings of the National Academy of Sciences of the United States of America 108, 1349-1354 (2011).

63. Choudhury, H.G. et al. Structure of an antibacterial peptide ATP-binding cassette transporter in a novel outward occluded state. Proceedings of the National Academy of Sciences of the United States of America 111, 9145-9150 (2014).

64. Dawson, R.J.P. \& Locher, K.P. Structure of the multidrug ABC transporter Sav1866 from Staphylococcus aureus in complex with AMP-PNP. Febs Letters 581, 935-938 (2007).

65. Ramachandra, M. et al. Human P-glycoprotein exhibits reduced affinity for substrates during a catalytic transition state. Biochemistry 37, 5010-5019 (1998).

66. Perez, C. et al. Structure and mechanism of an active lipid-linked oligosaccharide flippase. Nature 524, 433-+ (2015).

67. Kim, J.M. et al. Subnanometre-resolution electron cryomicroscopy structure of a heterodimeric ABC exporter. Nature 517, 396-U598 (2015).

68. Oldham, M.L. et al. A mechanism of viral immune evasion revealed by cryo-EM analysis of the TAP transporter. Nature 529, 537-40 (2016).

69. Mishra, S. et al. Conformational dynamics of the nucleotide binding domains and the power stroke of a heterodimeric ABC transporter. Elife 3(2014).

70. Zutz, A. et al. Asymmetric ATP Hydrolysis Cycle of the Heterodimeric Multidrug ABC Transport Complex TmrAB from Thermus thermophilus. Journal of Biological Chemistry 286, 7104-7115 (2011).

71. Mittal, A., Bohm, S., Grutter, M.G., Bordignon, E. \& Seeger, M.A. Asymmetry in the Homodimeric ABC Transporter MsbA Recognized by a DARPin. Journal of Biological Chemistry 287, 2039520406 (2012).

72. Zaitseva, J. et al. A structural analysis of asymmetry required for catalytic activity of an ABCATPase domain dimer. Embo Journal 25, 3432-3443 (2006). 
73. Muallem, D. \& Vergani, P. ATP hydrolysis-driven gating in cystic fibrosis transmembrane conductance regulator. Philosophical Transactions of the Royal Society B-Biological Sciences $\mathbf{3 6 4}$, 247-255 (2009).

74. Grossmann, N. et al. Mechanistic determinants of the directionality and energetics of active export by a heterodimeric ABC transporter. Nature Communications 5(2014).

75. Jones, P.M. \& George, A.M. A reciprocating twin-channel model for ABC transporters. Quarterly Reviews of Biophysics 47, 189-220 (2014).

76. Wang, B., Dukarevich, M., Sun, E.I., Yen, M.R. \& Saier, M.H. Membrane Porters of ATP-Binding Cassette Transport Systems Are Polyphyletic. Journal of Membrane Biology 231, 1-10 (2009).

77. Zheng, W.H. et al. Evolutionary relationships of ATP-Binding Cassette (ABC) uptake porters. Bmc Microbiology 13(2013).

78. Husada, F. et al. Watching conformational dynamics of $A B C$ transporters with single-molecule tools. Biochemical Society Transactions 43, 1041-1047 (2015).

79. van Wonderen, J.H. et al. The central cavity of ABCB1 undergoes alternating access during ATP hydrolysis. Febs Journal 281, 2190-2201 (2014).

80. Stockner, T., Mullen, A. \& MacMillan, F. Investigating the dynamic nature of the ABC transporters: $A B C B 1$ and MsbA as examples for the potential synergies of MD theory and EPR applications. Biochemical Society Transactions 43, 1023-1032 (2015).

81. Gu, R.X. et al. Conformational Changes of the Antibacterial Peptide ATP Binding Cassette Transporter McjD Revealed by Molecular Dynamics Simulations. Biochemistry 54, 5989-5998 (2015).

82. Moradi, M. \& Tajkhorshid, E. Mechanistic picture for conformational transition of a membrane transporter at atomic resolution. Proceedings of the National Academy of Sciences of the United States of America 110, 18916-18921 (2013).

83. Willis, L.M. \& Whitfield, C. Structure, biosynthesis, and function of bacterial capsular polysaccharides synthesized by $A B C$ transporter-dependent pathways. Carbohydrate Research 378, 35-44 (2013).

84. Holland, I.B., Schmitt, L. \& Young, J. Type 1 protein secretion in bacteria, the ABC-transporter dependent pathway (Review). Molecular Membrane Biology 22, 29-39 (2005).

85. Kuhlbrandt, W. The Resolution Revolution. Science 343, 1443-1444 (2014).

86. Procko, E., O'Mara, M.L., Bennett, W.F.D., Tieleman, D.P. \& Gaudet, R. The mechanism of ABC transporters: general lessons from structural and functional studies of an antigenic peptide transporter. Faseb Journal 23, 1287-1302 (2009).

87. Rees, D.C., Johnson, E. \& Lewinson, O. ABC transporters: the power to change. Nature Reviews Molecular Cell Biology 10, 218-227 (2009).

88. Wilkens, S. Structure and mechanism of ABC transporters. F1000prime reports 7, 14 (2015).

89. ter Beek, J., Guskov, A. \& Slotboom, D.J. Structural diversity of ABC transporters. Journal of General Physiology 143, 419-435 (2014).

90. Zhang, P. Structure and mechanism of energy-coupling factor transporters. Trends in Microbiology 21, 652-659 (2013).

91. Jin, M.S., Oldham, M.L., Zhang, Q.J. \& Chen, J. Crystal structure of the multidrug transporter Pglycoprotein from Caenorhabditis elegans. Nature 490, 566-+ (2012).

92. Kodan, A. et al. Structural basis for gating mechanisms of a eukaryotic P-glycoprotein homolog. Proceedings of the National Academy of Sciences of the United States of America 111, 40494054 (2014).

93. Lee, J.Y., Yang, J.G., Zhitnitsky, D., Lewinson, O. \& Rees, D.C. Structural Basis for Heavy Metal Detoxification by an Atm1-Type ABC Exporter. Science 343, 1133-1136 (2014).

94.Li, J.Z., Jaimes, K.F. \& Aller, S.G. Refined structures of mouse P-glycoprotein. Protein Science 23, 34-46 (2014)

95. Srinivasan, V., Pierik, A.J. \& Lill, R. Crystal Structures of Nucleotide-Free and Glutathione-Bound Mitochondrial ABC Transporter Atm1. Science 343, 1137-1140 (2014).

96. Ward, A., Reyes, C.L., Yu, J., Roth, C.B. \& Chang, G. Flexibility in the ABC transporter MsbA: Alternating access with a twist. Proceedings of the National Academy of Sciences of the United States of America 104, 19005-19010 (2007).

97. Locher, K.P. Review. Structure and mechanism of ATP-binding cassette transporters. Philosophical transactions of the Royal Society of London. Series B, Biological sciences 364, 23945 (2009). 
98.Borbat, P.P. et al. Conformational motion of the ABC transporter MsbA induced by ATP hydrolysis. Plos Biology 5, 2211-2219 (2007).

99. Loo, T.W., Bartlett, M.C. \& Clarke, D.M. Human P-glycoprotein is active when the two halves are clamped together in the closed conformation. Biochemical and Biophysical Research Communications 395, 436-440 (2010).

100. Xu, K. et al. Crystal structure of a folate energy-coupling factor transporter from Lactobacillus brevis. Nature 497, 268-+ (2013). 
Box 1: Architectures and conserved motifs of $A B C$ transporters

ABC transporters contain four core domains, two cytoplasmic NBDs and two TMDs. The NBDs point their conserved motifs towards each other, which generates two ATP-binding sites between the Ploops of one NBD and the signature motif of the other. In certain heterodimeric transporters (and in some full transporters), there are degenerate ATPase sites, where key sequence motifs carry mutations. Although nucleotide binding to these degenerate motifs may be preserved, hydrolysis at the modified ATPase sites is severely inhibited or abolished (reviewed in ${ }^{86}$ ).

The TMDs form a single, central pathway for their cognate substrate(s). Whereas NBDs are structurally similar, the folds of TMDs are diverse (reviewed in ${ }^{1,87,88}$ ). In bacterial $A B C$ importers, TMDs are expressed as separate protein subunits that either belong to type I or type II based on whether the fold is similar to that first observed in A. fulgidus ModBC ${ }^{41}$ (type 1) or E. coli BtuCD ${ }^{53}$ (type 2). Recently, ECF-type transporters have revealed another fold that can be counted to the ABC importer family. ECF-type transporters facilitate high-affinity uptake of certain vitamins into bacteria. Although shown in Fig. 1 for completeness, they will not be discussed here, and the reader is referred to recent reviews ${ }^{89,90}$. There is currently only one structurally defined fold of $A B C$ exporters, first revealed by the bacterial Sav1866 protein ${ }^{54}$ and subsequently found in all reported crystal structures of $A B C$ exporters ${ }^{34,55,57,58,63,66,91-96}$. It will be referred to as the B-family $A B C$ exporter fold. In this fold, the NBDs are fused to the C-termini of TMDs, which generates half-transporters that assemble to full transporters by forming homodimers or heterodimers. In some B-family ABC exporters, all four domains are fused to a single polypeptide (e.g. in ABCB1 or ABCB4).

An important observation was that although the three TMD folds discussed here show no structural similarity, they all feature a short, cytoplasmic helix roughly parallel to the membrane plane and facilitating critical contacts with the NBDs (Fig. 1b). Due to its key role transmitting ATP-driven conformational changes from the NDBs to the TMDs, it has been termed "coupling helix" ${ }^{154,97}$. Coupling helices allow distinct and evolutionarily unrelated TMD folds to harness the conformational power delivered by NBDs.

a

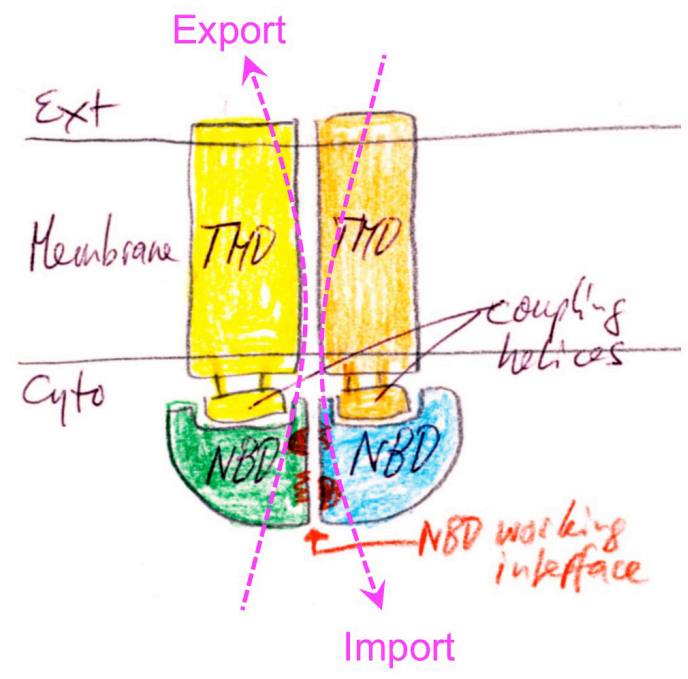

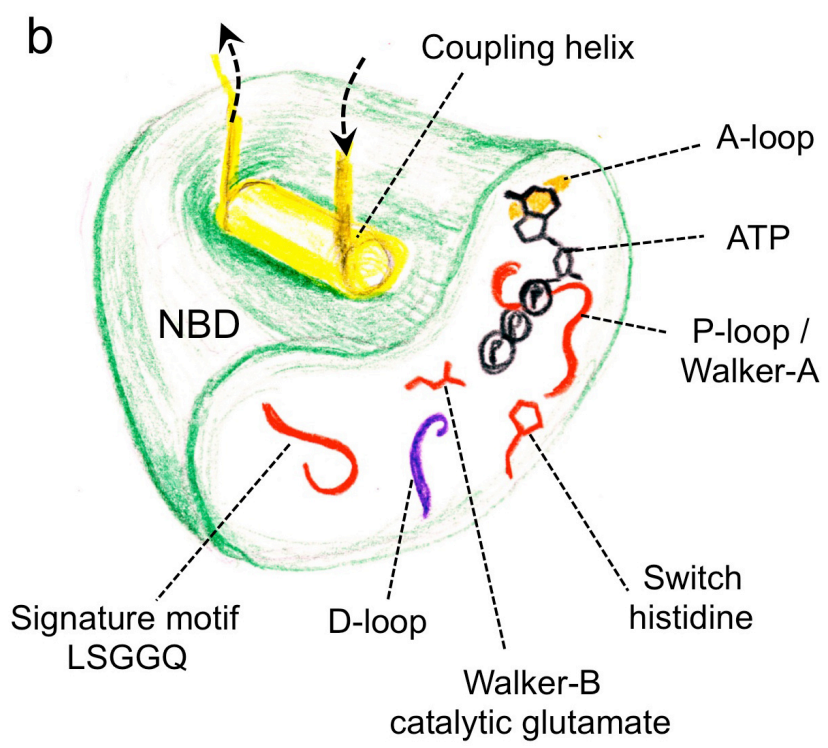

b catalytic glutamate 
Figure legend box 1: a, Domain arrangement of $A B C$ transporters: At the NBD interface, red halfcircles and lines indicate the $\mathrm{P}$-loops and $A B C$ signature motifs, respectively. Any given $A B C$ transporter is either an importer or an exporter. Coupling helices transmit conformational changes from the NBDs to the TMDs and vice versa. $\mathbf{b}$, Schematic of a single NBD. The location of the conserved and functionally critical motifs are indicated and labeled. They include the P-loop (or Walker-A motif) that binds the $\alpha$ - and $\beta$-phosphates of ATP, the A-loop that provides an aromatic side chain that packs against the purine ring of adenine, a Walker-B motif providing the catalytic glutamate, a signature LSGGQ motif that pins and orients ATP during hydrolysis, a "switch histidine" that stabilizes the transition state geometry, a Q-loop providing contacts to the TMD (not shown), and a dimerization or D-loop that has a role in coupling hydrolysis to transport (reviewed in ${ }^{87}$ ). A groove in the NBD surface forms the contact interface with the coupling helix of the TMD. While the coupling helix is not the only contact between TMDs and NBDs, it is the only architecturally conserved one among distinct TMD folds and provides the majority of contacts between domains. 
Box 2: Inward-facing conformations in B-family $A B C$ exporter structures

The physiological relevance of inward-open conformations with wide separation of the NBDs is controversially discussed. Those arguing in favor would point out that extreme conformational flexibility has been observed in certain transporters by DEER-EPR ${ }^{69,98}$. Furthermore, the exchange of ADP for ATP following a hydrolysis cycle requires an apo-state to be populated, even if only briefly. In addition, inward-facing conformations (and to some degree NBD separation) are required if alternating access is postulated, because an inward-facing cavity would form the substrate binding site. The only structures of B-family $A B C$ exporters with bound transport substrates are those of ScAtm 1 and the bacterial homologue NaAtm $1^{93,95}$. The two structures are very similar and revealed nucleotide-free, inward-facing conformations with substrates (GSH and GSSG) bound to an inwardfacing cavity at the level of the inner membrane leaflet. In both structures, however, there is physical contact between the NBDs, afforded through long helices at the C-terminal ends of the NBDs.

Those arguing against the functional relevance argue that the absence of nucleotides or the presence of ADP alone used in structural and spectroscopic studies do not reflect physiological conditions, because the cytoplasm of viable cells contains multi-millimolar concentrations of ATP, as well as ADP at about a tenth of the ATP concentration. Apo-states or ADP-states are therefore likely short-lived and transient in vivo. Furthermore, many B-family exporters require a lipid bilayer for substratestimulated ATP hydrolysis, whereas only low (and often non-stimulated) ATPase rates are observed in detergent. Moreover, P-glycoprotein has been found to be functional even when its two halves were cross-linked and a wide, inward-facing conformation as observed in crystal structures was prevented ${ }^{99}$. Also, separating the NBDs has been demonstrated to inhibit the activity of B-family ABC exporters: $A B C B 1$ is inhibited by a nanobody between the $\mathrm{NBDs}^{34}$ while TAP is inhibited by a viral peptide wedged between the TMDs ${ }^{68}$, also preventing NBD dimer closure. The LLO flippase PgIK is currently the only B-family exporter crystallized in two distinct apo-states, revealing inward-facing conformations and NBD separations that differ in distance and relative orientation ${ }^{66}$. This argues against a functional relevance of the, inward-facing conformations. Rather, lattice contacts and detergent effects can dictate the degree of NBD separation. 
Figure 1

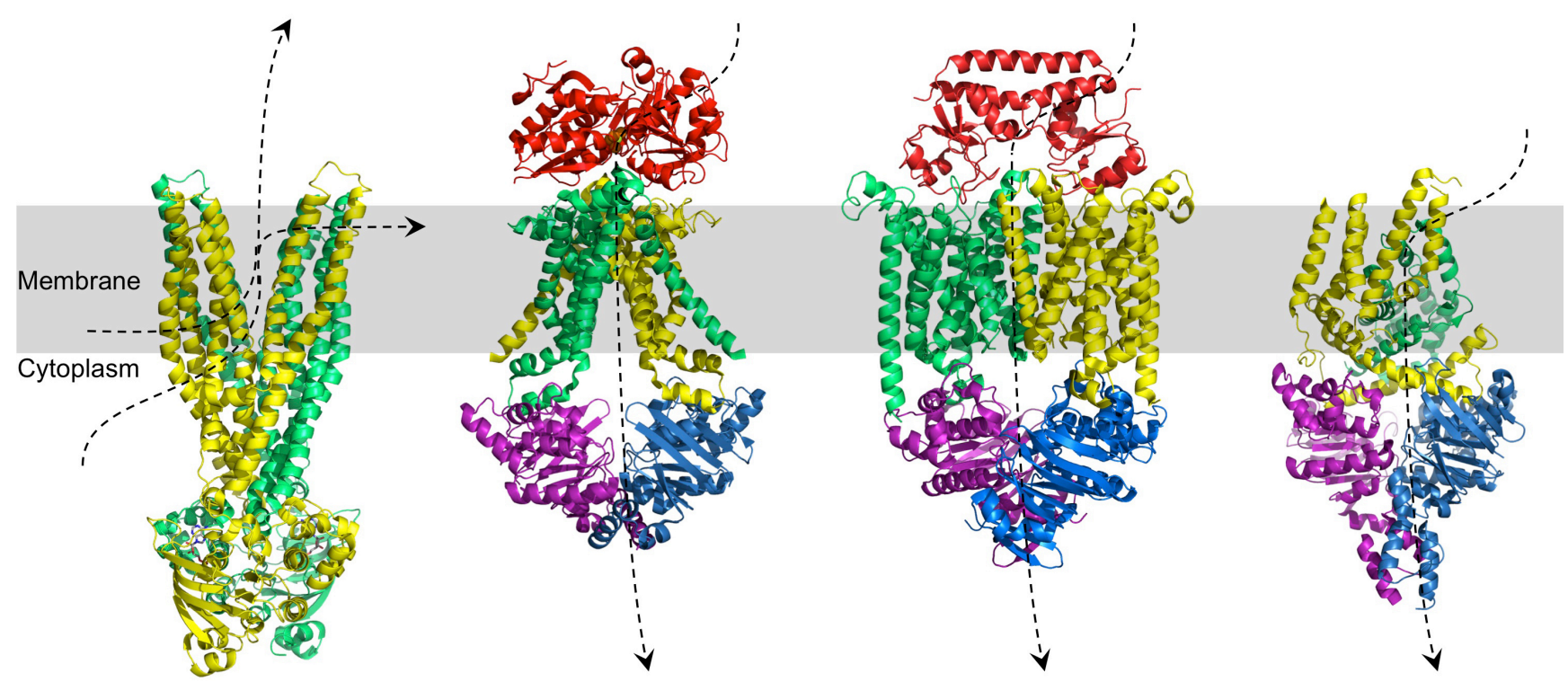

Figure 1: $A B C$ transporter structures. The molecules shown in the figure defined the TMD folds of the indicated sub-families: Staphylococcus aureus multidrug transporter Sav1866 ${ }^{54}$ (B-family $A B C$ exporters); Archaeoglobus fulgidus molybdate / tungstate transporter ModBC-A ${ }^{41}$ (type 1 ABC importer); Escherichia coli vitamin B12 transporter BtuCD-F ${ }^{53}$ (type 2 ABC importer); Lactobacillus brevis folate importer EcfAST ${ }^{100}$ (ECF-type $A B C$ importer). TMDs are colored yellow and green, periplasmic substrate binding proteins are in red, NBDs in $A B C$ importers are purple and blue. Note that the TMDs and NBDs are fused in B-family exporters. Whereas bacterial B-family ABC exporters are homodimeric or heterdimeric, many eukaryotic homologs contain all four domains on a single polypeptide chain. Dashed arrows indicate the known or suspected substrate paths during translocation. One of the TMDs in ECF-transporters is variable, and there are no coupling helices similar to those of canonical $A B C$ transporters. 
Figure 2

a

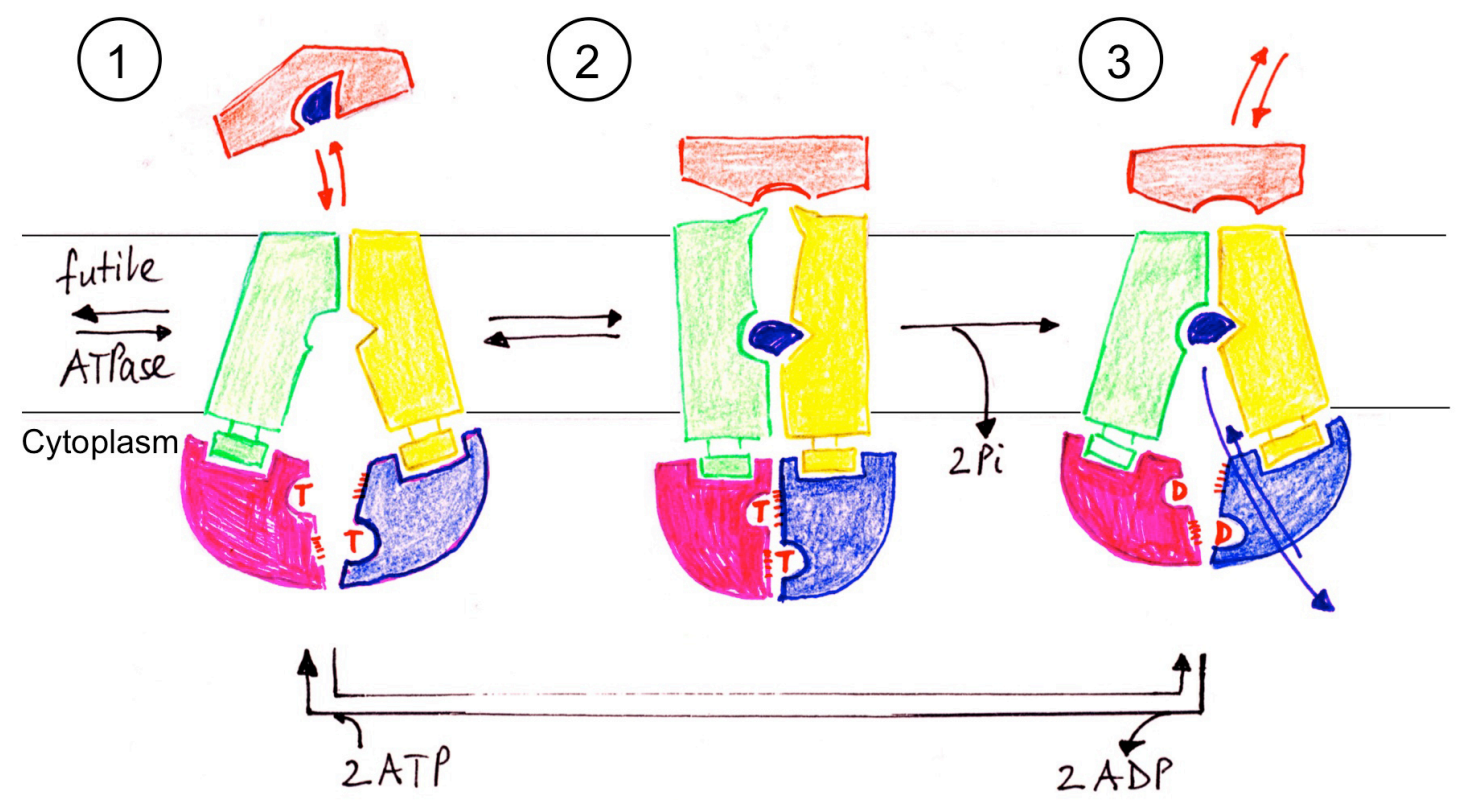

b

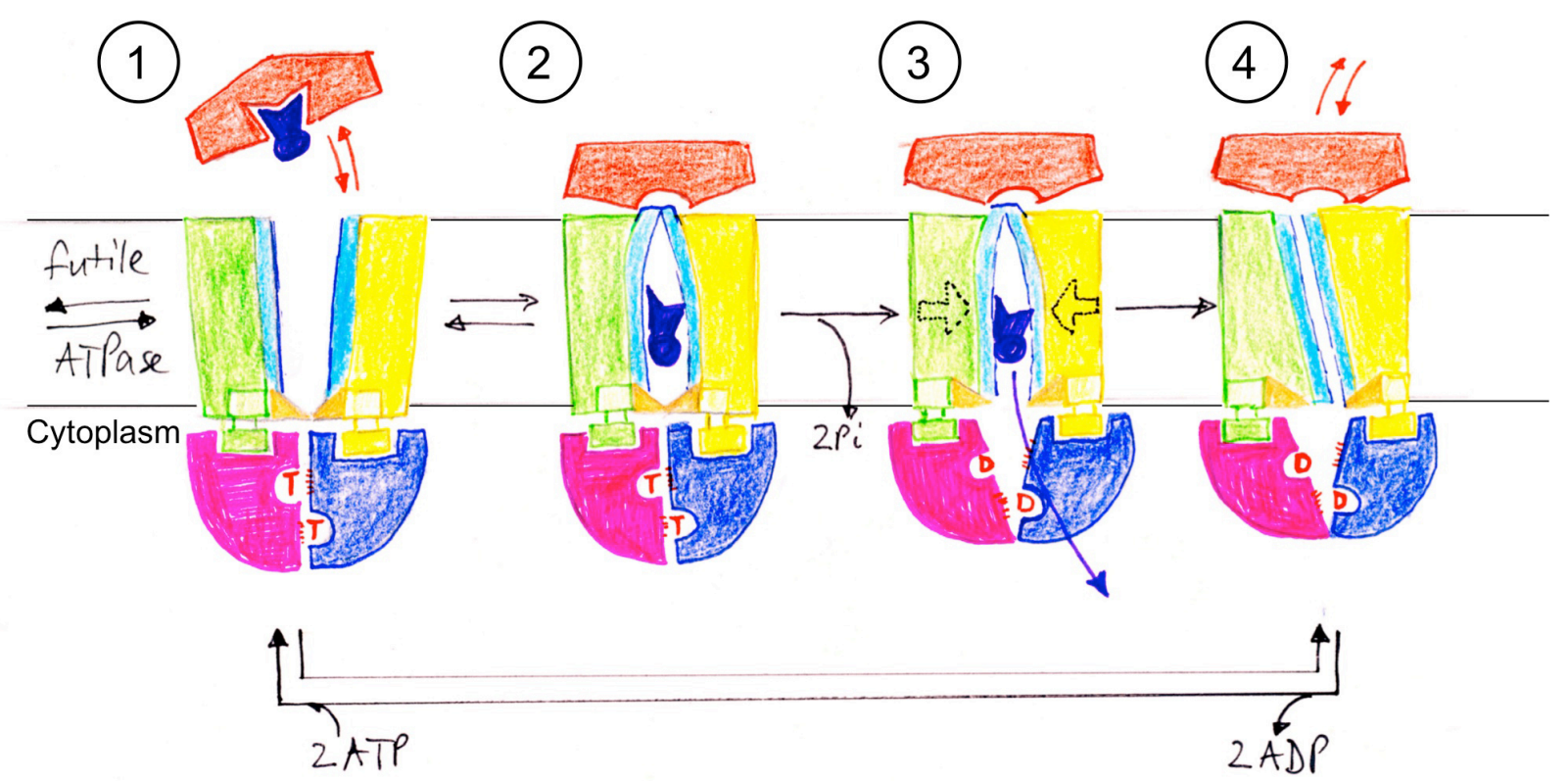

Figure 2. Distinct mechanisms of type 1 and type 2 ABC importers. TMDs are colored green and yellow, NBDs are colored pink and purple, binding proteins are colored orange, with substrates in dark blue. "T" and "D" denote ATP and ADP, respectively. Dotted red lines in the NBDs depict the $A B C$ signature motifs. Numbers above schematics indicate states. See text for detailed explanations. a, Schematic of a type 1 importer importer mechanism. $\mathbf{b}$, Schematic of a type 2 importer containing three distinct gates. The blue lines in the TMDs represent TM5 forming cyto-gate 1 and the peri-gate, whereas the orange triangles represent cyto-gate 2, formed by the loop between TM2 and TM3. Small arrows in TMDs of state 3 indicate forces acting on the translocation pathway and entrapped substrate. 
Figure 3

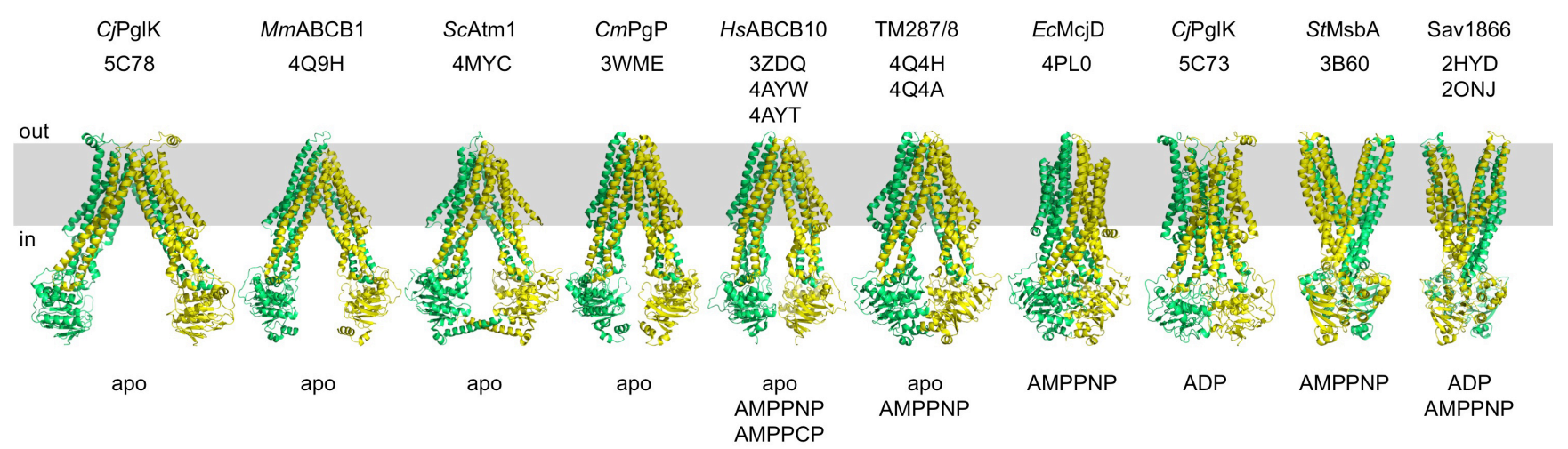

Figure 3: Conformations in selected B-family $A B C$ exporter structures. Species abbreviations and protein names are shown in the top row, relevant pdb codes above the ribbon diagrams, bound nucleotides below. "In" refers to the cytoplasmic side of the membrane. If multiple nucleotide states are indicated (e.g. apo and AMPPNP), the relevant structures did not differ significantly in terms of NBD separation. 
Figure 4

a

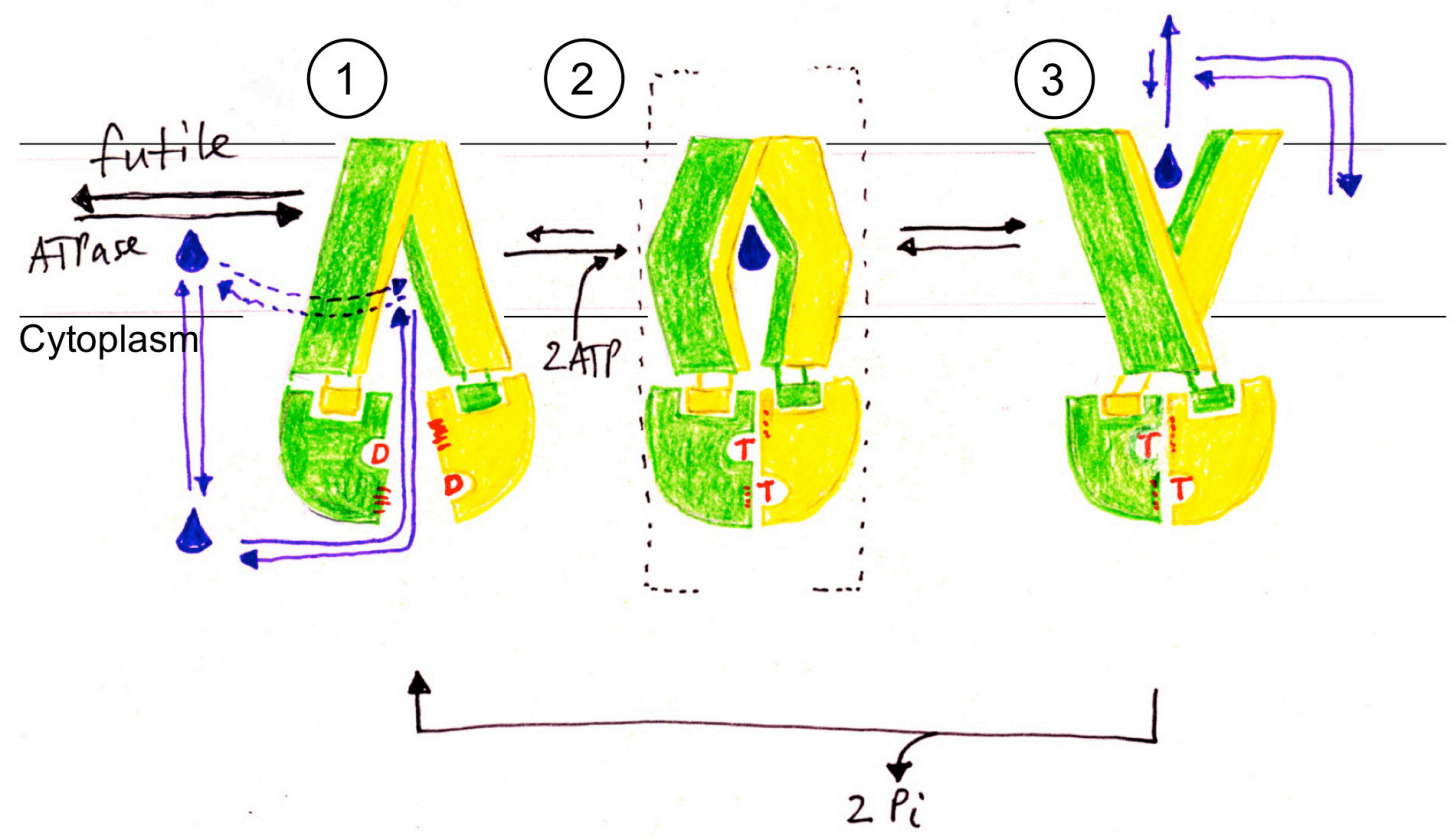

b

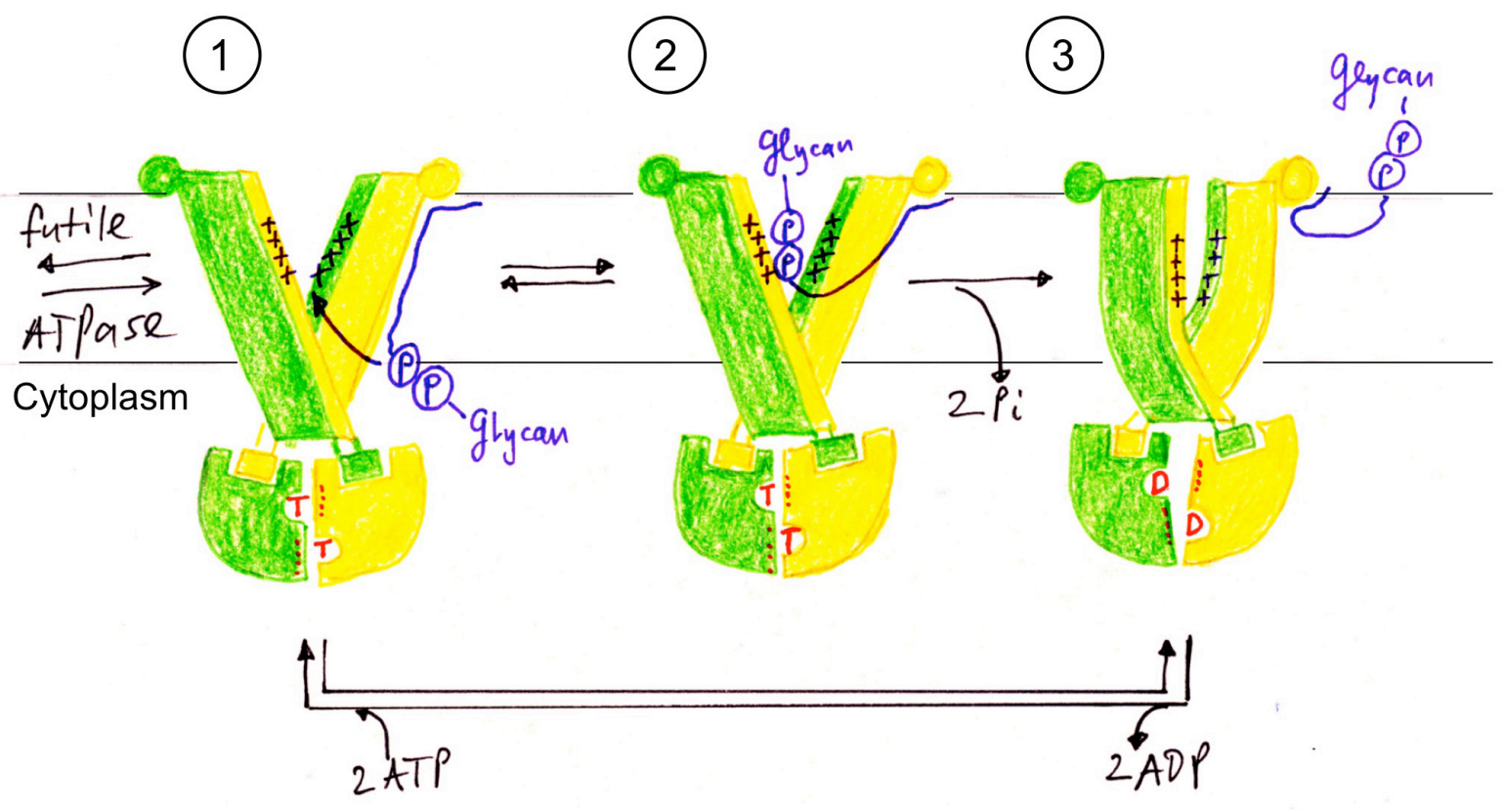

Figure 4: Distinct mechanisms of B-family $A B C$ exporters. Half-transporters are colored green and yellow. "T" and "D" denote ATP and ADP, respectively. Dotted red lines in the NBDs depict the ABC signature motifs. Circled numbers denote states. In outward-open conformations, the TMDs of the transporters form two wings towards the external side of the membrane. Each wing contains two TM helices from one TMD and four TM helices from the other. See text for details. a, Alternating access proposed for multidrug or peptide transporters. b, Outward-only mechanism with substrate loading directly into outward-facing cavity as proposed for LLO flippase PglK. 